\title{
APPLICATION OF HOMOGENIZATION THEORY TO THE STUDY OF TRABECULAR BONE MECHANICS
}

\author{
S. J. Hollister,* D. P. Fyhrie, $†$ K. J. Jepsen and S. A. Goldstein \\ Orthopaedic Research Laboratories, Section of Orthopaedic Surgery, \\ The University of Michigan, Ann Arbor, MI 48109, and † Bone and Joint Center, Henry Ford Hospital, \\ Detroit, MI 48202, U.S.A.
}

\begin{abstract}
It is generally accepted that the strength and stiffness of trabecular bone is strongly affected by trabecular microstructure. It has also been hypothesized that stress induced adaptation of trabecular bone is affected by trabecular tissue level stress and/or strain. At this time, however, there is no generally accepted (or easily accomplished) technique for predicting the effect of microstructure on trabecular bone apparent stiffness and strength or estimating tissue level stress or strain. In this paper, a recently developed mechanics theory specifically designed to analyze microstructured materials, called the homogenization theory, is presented and applied to analyze trabecular bone mechanics. Using the homogenization theory it is possible to perform microstructural and continuum analyses separately and then combine them in a systematic manner. Stiffness predictions from two different microstructural models of trabecular bone show reasonable agreement with experimental results, depending on metaphyseal region, $\left(R^{2}>0.5\right.$ for proximal humerus specimens, $R^{2}<0.5$ for distal femur and proximal tibia specimens). Estimates of both microstructural strain energy density (SED) and apparent SED show that there are large differences (up to 30 times) between apparent SED (as calculated by standard continuum finite element analyses) and the maximum microstructural or tissue SED. Furthermore, a strut and spherical void microstructure gave very different estimates of maximum tissue SED for the same bonc volume fraction (BV/TV). The estimates from the spherical void microstructure are between 2 and 20 times greater than the strut microstructure at $10-20 \%$ BV/TV.
\end{abstract}

\section{INTRODUCTION}

Stress and strain fields in individual trabeculae are determined by both overall metaphyseal geometry and the microstructural organization of a local region of trabeculae. However, directly calculating stress and strain fields for each trabecula of a whole metaphysis is impossible with current computational technology. Current techniques for determining trabecular stress have focused on either analyzing very small regions of bone with a limited number of trabeculae, or analyzing a much larger region of bone assuming it to be a solid with apparent material properties. Neither technique provides direct information about trabecular tissue stresses and strains in an entire metaphyseal region. The homogenization theory is a recently developed method which provides a systematic way to combine stress analyses at the metaphyseal and individual trabecula level. This paper presents the initial application of homogenization theory to the study of trabecular bone mechanics.

Microstructural analyses are generally used to predict apparent moduli and stress distributions within representative pieces of a given microstructure. These analyses assume that the whole microstructure within a material is constructed by repeating the representative piece analyzed. This type of analysis is quite

Received in final form 14 February 1991.

*Author to whom correspondence should be addressed: Orthopaedic Research Laboratories, Room G-0161, 400 N. Ingalls Building, Ann Arbor, MI 48109, U.S.A. common in the cellular plastics field (see, for example, Hilyard, 1982; Gibson and Ashby, 1988; Patel and Finnie, 1970; Ko, 1965; Gent and Thomas, 1959). To calculate apparent moduli, these analyses relate average stress fields to average strain fields calculated under assumptions about in situ boundary conditions on the representative section of microstructure. The choice of in situ boundary conditions (for example, choosing uniform displacements or uniform stress) will greatly affect the resulting apparent stiffness prediction (Hill, 1963; Suquet, 1985; Beaupre and Hayes, 1985). An assumption of in situ boundary conditions is also used to predict stress distributions within the microstructure and possible failure modes (Luxmoore and Owen, 1982; Gibson and Ashby, 1988).

Microstructural analyses of trabecular bone have followed the general approach used in the cellular plastics field. McElhaney et al. (1970) developed a porous block model of trabecular bone based on integration of spring stiffness loaded in parallel or in series. Using this model, they found good agreement between predictions of apparent stiffness and experimentally measured stiffness values in the skull diplö layer. Pugh et al. (1973) modeled the subchondral trabecular bone as a collection of structural plates and concluded that bending and buckling were major modes of deformation of the trabeculae. Williams and Lewis (1982) modeled the exact structure of a twodimensional section of trabecular bone with plane strain finite elements to predict the apparent transversely isotropic elastic constants. They concluded that the modulus of trabecular bone tissue was much less than that of cortical bone tissue. Gibson (1985) 
developed general models of trabecular bone structure using analytical techniques for porous solids. She predicted the dependence of apparent stiffness and strength on apparent density for different structural types of trabecular bone. Gibson found either a linear or square dependence of the apparent modulus on density depending on the type of trabecular deformation. Beaupre and Hayes (1985) developed a threedimensional spherical void model of trabecular bone and used finite element analyses to predict apparent stiffness and strength, as well as the stress distribution within the trabeculae. They found reasonable agreement between their predictions and experimental values. These analyses have provided much insight into the microstructural mechanics of trabecular bone. However, the lack of a systematic way to use models of trabecular structure for the analysis of whole metaphyseal regions has limited the application of microstructural analyses.

In orthopaedic biomechanics, most stress analyses of metaphyseal regions have modeled trabecular bone as a solid continuum with apparent mechanical properties (for a review, see Huiskes and Chao, 1983). While some investigators have introduced modifications of apparent stress predictions to approximate trabecular tissue stresses (Carter et al., 1987), no direct estimate of trabecular tissue stress for models of large regions of bone have been presented. In this paper, the homogenization theory is used to obtain estimates of individual trabeculae stress and strain states for large regions of cancellous bone. This development is significant in that most stress affected biological processes, such as bone remodeling, and many mechanical processes, such as trabecular bone fatigue, are most likely governed by trabecular tissue stresses and strains, rather than the average stresses and strains which can be calculated using standard finite element approaches. Standard continuum finite element analyses cannot estimate trabecular tissue stresses and strains for large regions of cancellous bone due to computational limitations. The homogenization theory eliminates this barrier, providing the means to directly estimate trabecular stress and strain patterns for large regions of cancellous bone.

The work presented here is the first application of the homogenization method to the analysis of trabecular bone mechanics. First, the background of homogenization theory will be presented and the theory will be compared with existing methods used for microstructural analysis of trabecular bone. Second, the predictions of homogenization analysis will be validated with analytical and numerical benchmarks. Third, apparent stiffness predictions from different models of trabecular bone specimens will be compared with experimentally measured values from the same specimens. Finally, continuum stress and microstructural stress predictions from three different models of trabecular bone will be compared for a model of trabecular bone loaded by a metal peg.

\section{Notation}

In this paper, microstructural analyses or measured quantities (those specifically dealing with an individual trabecula) will be denoted as 'tissue' level analyses or quantities. Analyses or quantities which assume trabecular bone act as a solid continuum and will be denoted as 'apparent' level analyses or quantities. For example, tissue stiffness will refer to the stiffness of the material composing an individual trabecula, while apparent stiffness will refer to the stiffness of a cube of bone containing many trabeculae.

\section{BACKGROUND}

The homogenization theory is specifically designed to analyze the physics of microstructured materials (Bensoussan et al., 1978; Sanchez-Palencia, 1980). It has been used extensively to analyze composite materials (Suquet, 1985; Guedes, 1990), and also in predicting optimal topology of microstructured materials (Bendsoe and Kikuchi, 1988; Lurie et al., 1982). In biomechanics, Crolet et al. (1988, 1990) applied the homogenization theory to model cortical bone mechanics. They predicted anisotropic elastic constants which were close to experimental results using a model of two osteons. Hollister et al. (1989) applied homogenization analysis to trabecular bone mechanics and found that the power law fit between volume fraction and continuum stiffness was very dependent on the microstructural geometry.

All microstructural analysis methods use assumptions about the microstructure to make the analysis tractable. The homogenization theory makes two specific assumptions which differ from those made by other methods. First, the total tissue strain is separated into an apparent strain plus a fluctuating component (Suquet, 1985):

$$
\left\{\varepsilon_{\text {tissue }}\right\}=\left\{\varepsilon_{\text {app }}\right\}+\left\{\varepsilon^{*}\right\},
$$

where $\left\{\varepsilon_{\text {issue }}\right\}$ is the total tissue strain, $\left\{\varepsilon_{\mathrm{app}}\right\}$ is the apparent strain which varies only on a macroscopic level, and $\left\{\varepsilon^{*}\right\}$ is a fluctuating component which varies on both the macroscopic and microscopic levels Second, the microstructure is assumed to be locally periodic (Sanchez-Palencia, 1980), which means that $\left\{\varepsilon^{*}\right\}$ will be periodic on the microstructural scale. Local periodicity means that the microstructure is periodic at all points in the macroscopic body, but the nature of the periodicity and the geometry of the microstructure itself may vary from point to point on the macroscopic scale. The resulting variational form of the homogenization microstructural equilibrium equation is:

$$
\begin{aligned}
& \int_{V_{\text {cell }}}\{\varepsilon(v)\}^{T}[C]\left\{\varepsilon^{*}\right\} \mathrm{d} V_{\text {cell }} \\
& =-\int_{V_{\text {coll }}}\{\varepsilon(v)\}^{T}[C]\left\{\varepsilon_{\text {app }}\right\} \mathrm{d} V_{\text {cell }},
\end{aligned}
$$


where $\{\varepsilon(v)\}$ is the virtual strain vector, $[C]$ is the tissue stiffness matrix, $V_{\text {cell }}$ is the volume of the microstructural model, $\left\{\varepsilon^{*}\right\}$ is the fluctuating strain component, and $\left\{\varepsilon_{\text {app }}\right\}$ is the apparent strain component.

In general, $\left\{\varepsilon_{\text {app }}\right\}$ from equation (2) is not known a priori. Since equation (2) is linear, however, any apparent strain state $\left\{\varepsilon_{\mathrm{app}}\right\}$ can be represented by the linear superposition of other strains. By choosing $\left\{\varepsilon_{\text {app }}\right\}$ to be a linear superposition of unit $x$ normal, $y$ normal, and $x y$ shear strains, (chosen as compressive and negative by convention; six unit strain components are used for three-dimensional analysis), equation (2) can be rewritten as (see Appendix A for details):

$$
\begin{aligned}
\int_{V_{\text {coll }}}\{\varepsilon(v)\}^{T}[C]\left\{\varepsilon^{k *}\right\} \mathrm{d} V_{\text {cell }} \\
=\int_{V_{\text {coll }}}\{\varepsilon(v)\}^{T}\left\{C^{k}\right\} \mathrm{d} V_{\text {cell }},
\end{aligned}
$$

where $\left\{C^{k}\right\}$ is the $k$ th column of the stiffness matrix, $\left\{\varepsilon^{k *}\right\}$ is the $k$ th fluctuating strain component corresponding to $\left\{C^{k}\right\}$, and the other terms are defined previously. Solving equation (3) for each column of the stifiness matrix and combining the results with equation (1) allows the total tissue strain to be directly calculated from any apparent strain state as:

$$
\begin{aligned}
\left\{\varepsilon_{\text {issue }}\right\} & =[M]\left\{\varepsilon_{\text {app }}\right\} \\
{[M] } & =[I]-\left[\left\{\varepsilon^{* k}\right\}\right],
\end{aligned}
$$

where here we denote $[M]$ as the local structure matrix $\dagger$ since it represents the effect of the microstructure on the tissue strain, [I] is the identity matrix, and $\left[\left\{\varepsilon^{* k}\right\}\right]$ is a matrix with columns composed of the fluctuating strain components $\left\{\varepsilon^{* k}\right\}$ and the other terms are as defined previously. The fluctuating component $\left\{\varepsilon^{*}\right\}$ is independent of $\left\{\varepsilon_{\mathrm{app}}\right\}$ and is determined only by the local periodicity requirement and the geometry and stiffness of the microstructure.

The apparent stiffness matrix can then be calculated as:

$$
\left[C_{\text {app }}\right]=\frac{1}{V_{\text {cell }}} \int_{V_{\text {cell }}}[C][M] \mathrm{d} V_{\text {cell }},
$$

where $\left[C_{\mathrm{app}}\right]$ is the apparent stiffness matrix and the other terms are as defined previously.

Standard mechanics approaches, which have been applied to analyze composite materials (Hill, 1963; Hashin, 1962; reviewed by Hashin, 1983) and cellular foams (Gibson and Ashby, 1988; Huber and Gibson, 1988; Christenson, 1986; Gibson and Ashby, 1982; Iremonger and Lawler, 1980; Kanakkanatt, 1973; Lederman, 1971; Patel and Finnie, 1970; Ko, 1965; Gent and Thomas, 1959) as well as trabecular bone (Beaupre and Hayes, 1985; Gibson and Ashby, 1988;

† Suquet (1985) denotes the tensor equivalent of $[M]$ as the strain localization tensor.
Gibson, 1985; Williams and Lewis, 1982; Pugh et al., 1973), generally assume that the effect of the apparent stress, $\left\{\sigma_{\text {app }}\right\}$ (or strain, $\left\{\varepsilon_{\text {app }}\right\}$ ) on the microstructure can be represented by self equilibrated anti-periodic traction or displacement boundary conditions. These approaches then solve for the total tissue strain using the standard form of the variational equilibrium equation (shown here for the traction boundary condition):

$$
\begin{aligned}
\int_{V_{\text {cell }}}\{\varepsilon(v)\}^{T}[C] & \left\{\varepsilon_{\text {tissue }}^{k}\right\} \mathrm{d} V_{\text {cell }} \\
& =\int_{S_{\text {cell }}}\{v\}^{T}\left\{t^{k}\right\} \mathrm{d} S_{\text {cell }},
\end{aligned}
$$

where $\{v\}$ is the virtual displacement vector, $\left\{t^{k}\right\}$ is the $k$ th traction vector (corresponding to unit $x$ normal, $y$ normal, $x y$ shear tractions), and $\left\{\varepsilon_{\text {tiseue }}^{k}\right\}$ is the $k$ th tissue strain resulting from the corresponding applied traction. Once $\left\{\varepsilon_{\text {tissue }}^{k}\right\}$ is determined, the apparent stiffness matrix and the relationship between $\left\{\varepsilon_{\mathrm{app}}\right\}$ and $\left\{\varepsilon_{\text {issue }}\right\}$ can be calculated. As noted by many authors (Hill, 1963; Hashin, 1962; Suquet, 1985), choosing a displacement boundary condition will provide an upper bound on the actual apparent stiffness while choosing a traction boundary condition will provide a lower bound on the actual apparent stiffness. The closeness of the two bounds is dependent on both the form of the boundary conditions and the microstructure being analyzed.

\section{METHODS}

The application of the homogenization theory to the analysis of trabecular bone mechanics was assessed both analytically and experimentally. First, the apparent stiffness predictions from the homogenization theory were compared with predictions from standard mechanics of material approaches. Second, the effect of the cell to region volume ratio was addressed by comparing the tissue strain state calculated using a direct finite element analysis and a homogenization analysis. Third, homogenization stiffness predictions from two different models of trabecular bone for 38 specimens were compared with experimentally measured stiffness values from the same specimens. Fourth, three different models of trabecular bone microstructure were used in a global model of a metal peg loading a cylindrical region of trabecular bone to assess the relationship between apparent and tissue strain energy density.

\section{Analytical assessment}

The homogenization stiffness predictions were compared with analytical predictions presented by Ishai and Cohen (1967) for a porous composite consisting of cubic inclusions. The analytical results were derived by applying both uniform stress and uniform displacement boundary conditions on the microstructure. By making simplifying assumptions about the stress and 
strain distribution, it was possible to derive the composite modulus by relating the average strain to the average stress (for details, see Counto, 1964; Ishai, 1965). The homogenization predictions were determined using a specially written finite element code to analyze models of the cubic void. This code solves equation (4) and uses the solution in equation (8) to compute the apparent stiffness matrix. The models consisted of 150 and 588 8-node brick elements (Fig. 1).

\section{Evaluation of cell size ratio effect on accuracy}

The effect of the cell size ratio on accuracy was assessed by analyzing a porous strut microstructure using standard finite element methods and comparing the result with an analysis using the homogenization theory. The strut microstructure was analyzed using a quarter symmetric model with two unit cells on a side. A compressive load was applied to one side of the model and the center of the model was constrained in the direction of the load. The model consisted of 2048 8-node brick elements and was analyzed using the MARC finite element code, (MARC Analysis Research Corporation, Palo Alto, CA). The model simulated a structure with four unit cells on one side loaded under uniform compression. The homogenization analysis involved analyzing one structural cell with the specially written finite element code to compute the local structure and apparent stiffness matrices. Following this, a solid continuum model with the apparent stiffness matrix was loaded with an equivalent pressure loading. The apparent strain from this model was post-processed with the local structure matrix to calculate the strain in the microstructure which was then compared with the direct estimate.

\section{Experimental evaluation}

The homogenization theory was experimentally evaluated by comparing stiffness predictions from two models of trabecular bone microstructure with experimentally measured stiffness values of 38 cubes from the proximal tibia, proximal humerus, and distal femur. The homogenization analysis was performed using the specially written finite element code. Experimental data were taken from the study of Ciarelli $e t$ al. (1986) and consisted of three orthogonal moduli from tests on $6-8 \mathrm{~mm}$ bone cubes. The tissue was assumed to be isotropic with a modulus of $5 \mathrm{GPa}$ (Choi et al., 1990; Kuhn et al., 1989; Mente and Lewis, 1989) and a Poisson's ratio of 0.3 in each analysis. In addition, the average trabecular morphology and anisotropy of the specimens were measured using a special three-dimensional micro CT scanner (Feldkamp et al., 1989; Goulet et al., 1989).

Two unit cell models of regular geometry were used to model the trabecular bone microstructure. These models included a strut model (Fig. 2) and a closed cubic void model, similar to that used in the analytical study (Fig. 1). All of these models were cubically symmetric. These models were constructed to match the bone volume fraction (BV/TV) and bone surface to bone volume ratio (BS/BV) by adjusting the inner void and outer cell dimension. As a further refinement, some of the strut and closed cell models were 'stretched' so that the cell axes ratios matched the experimentally measured mean intercept length (MIL) ratios. These cubes possessed structurally orthotropic symmetry. The equations relating cell dimension to morphology are given in Appendix B. In addition, the calculated apparent stiffness matrix [ $\left.C_{\text {app }}\right]$ was transformed from the principal trabecular coordinate system to the coordinate system in which the orthogonal

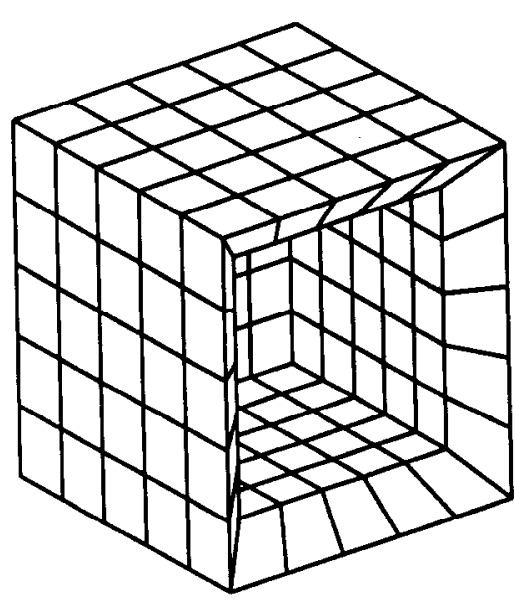

125 Element Mesh

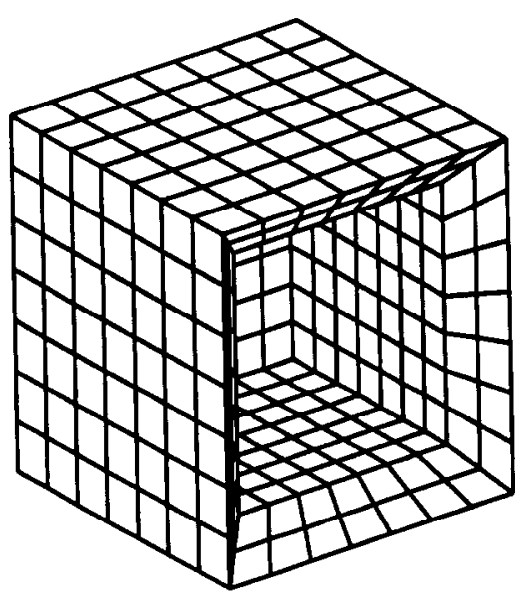

588 Element Mesh

Fig. 1. Finite element models used to analyze the cubical voids described by Ishai and Cohen (1967). Models shown are for $60 \%$ void fraction. The model on the left contains 150 elements and the model on the right contains 588 elements. 
moduli were measured. Thus, the microstructural models matched the principal trabecular orientation as well as the average morphology of the experimental specimens.

\section{Comparison of apparent and tissue strain energy density}

Comparisons of apparent stress and tissue stress parameters were made using a model of a metal peg loading trabecular bone. Nine analyses were performed for trabecular bone of 10,20 , and $30 \%$ volume fraction. Strut (similar to Fig. 2), spherical void (Fig. 3 ), and closed cell (similar to Fig. 1) structures were used to model trabecular bone microstructure. The specially written finite element code was used to

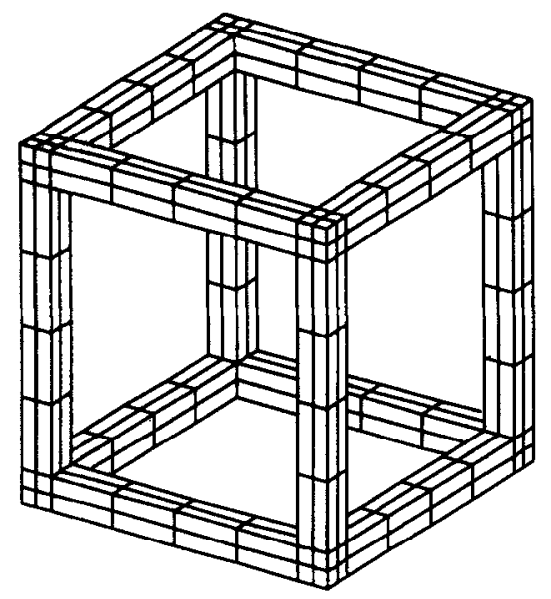

Fig. 2. Finite element model of the strut microstructural cell.

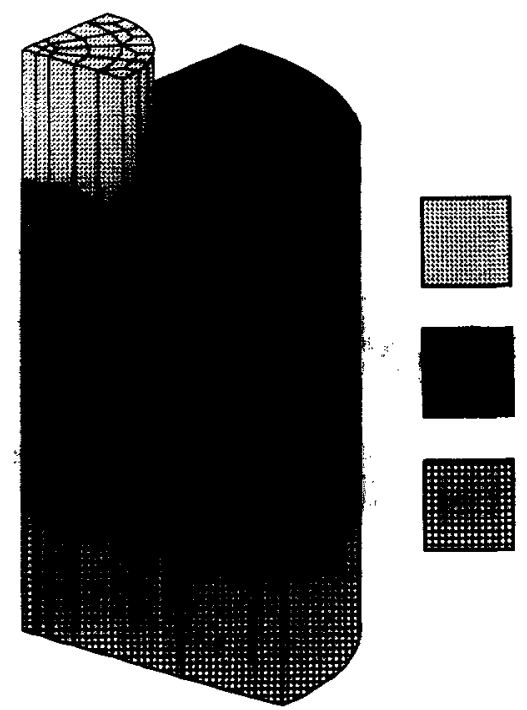

calculate the local structure matrix and apparent stiffness matrix for each analysis and the apparent stiffness matrix was input to the global finite element model of the continuum trabecular bone plus metal peg (Fig. 4). Quarter symmetry was assumed in the global model and a uniform pressure loading was applied to the top of the metal peg. The apparent strains were calculated using the finite element code MARC and post-processed with the local structure matrix to give the tissue strain estimate. Relationships between tissue and apparent strain energy density (SED) were assessed by dividing the peak tissue SED by the apparent SED and plotting the resulting ratio as a contour on the finite element mesh. In addition,

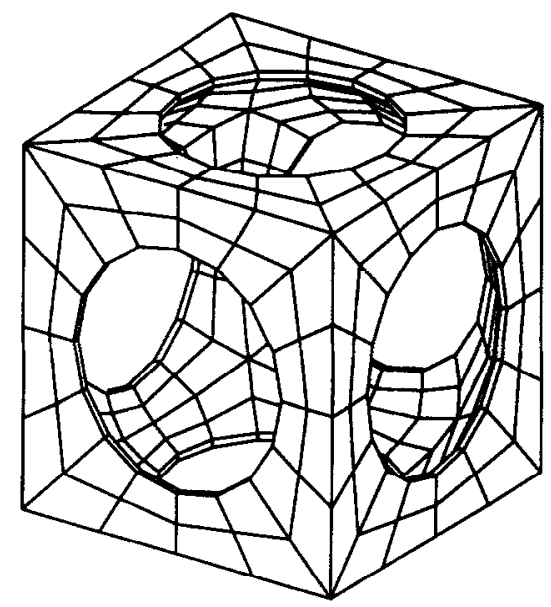

Fig. 3. Finite element model of the spherical void microstructural cell.

Metal

Bone/Homogenization Analysis

Isotropic Bone

Fig. 4. Global continuum model of a metal peg loading a region of trabecular bone. Quarter symmetry is assumed in the model, with the sides of the model constrained against out of plane displacements. Both the metal and isotropic bone were modeled as linear isotropic elastic materials with Young's modulus of $100 \mathrm{GPa}$ and $400 \mathrm{MPa}$, respectively. The bone from the homogenization analysis was modeled as an anisotropic elastic material with the anisotropic apparent stiffness computed from a homogenization analysis. 
the apparent SED was divided by the BV/TV to assess how well a modification of the apparent SED compared with the direct estimates of peak tissue SED.

\section{RESULTS}

\section{Analytical assessment}

Homogenization stiffness predictions and stiffness predictions calculated from uniform displacement and uniform traction boundary conditions (Ishai and Cohen, 1967) were compared for a porous composite with solid volume fraction between 10 and $60 \%$

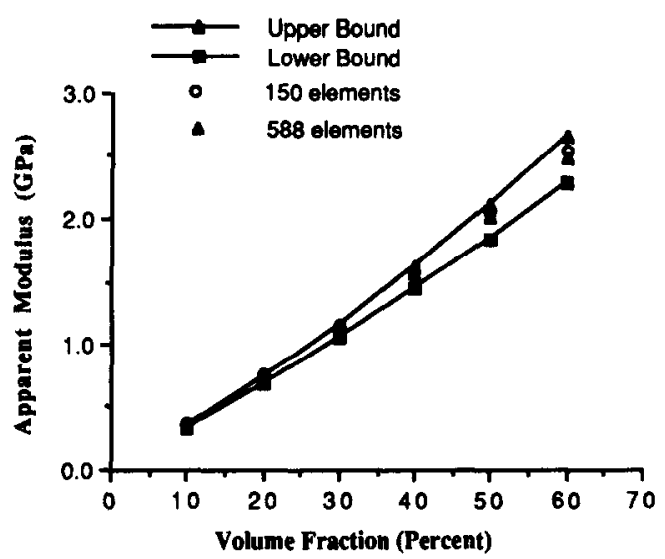

Fig. 5. Comparison of predicted moduli from homogenization analysis to analytically derived moduli from Ishai and Cohen (1967). Solid lines represent upper and lower bounds from analytical moduli. Open circles represent the moduli predicted from finite element homogenization analysis using 150 elements and open triangles represent moduli predicted from finite element homogenization analysis using 588 elements.
(Fig. 5). For volume fractions between 30 and $60 \%$, the homogenization prediction was between the uniform traction and uniform displacement predictions. At volume fractions less than or equal to $20 \%$, the homogenization predictions were greater than both uniform boundary condition predictions, which may be attributed in part to the finite element solution. The effect of the numerical solution on the prediction was examined by first refining the mesh followed by using selective reduced integration (Kikuchi, 1986). Reduced integration was applied because solid elements behave too stiffly if bending modes dominate, a situation which may be present in the low volume fraction composite with thin walls. Applying these techniques in our code did provide closer agreement with Ishai and Cohen's results.

\section{Evaluation of cell size ratio effect on accuracy}

The homogenization and direct estimates of stress distribution are compared in Fig. 6. One quarter of the exact model is shown on the left where the innermost cell is in the lower right-hand corner. The homogenization prediction for the innermost cell is shown on the right. The homogenization estimate is almost exact for the innermost cell, where boundary effects are the lowest. Nearer to the boundary where conditions violate theoretical assumptions, the errors are much larger. These results show that the homogenization theory will produce very accurate estimates of stress when the ratio of cell volume to regional volume is less than 0.0156 . This ratio corresponds to analyzing one structural cell for a locally periodic region of sixteen cells. Since the stress results for the innermost cell were nearly identical between the two methods, it follows that the apparent stiffness will be the same.
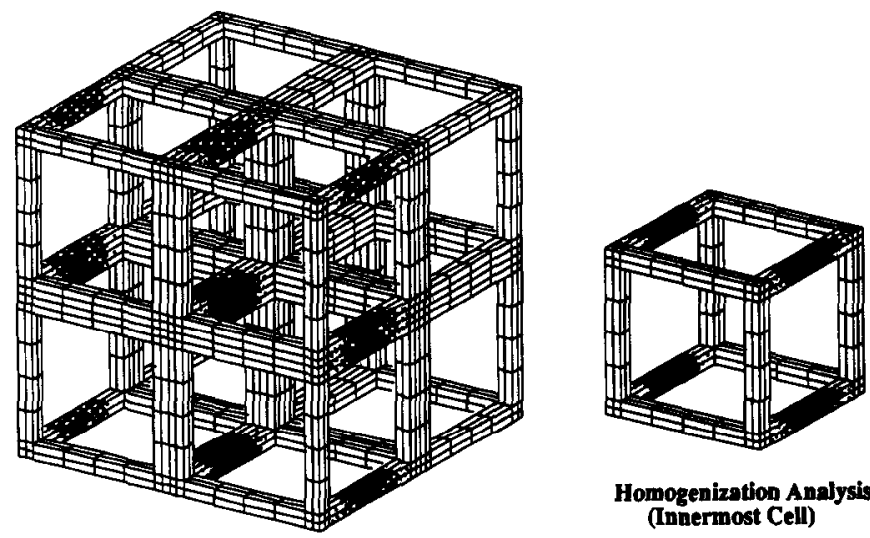

Von Mises Stress (MPa)

Standard Finite Element Analysis

Fig. 6. Von Mises stress distribution from standard finite element analysis and homogenization analysis for a regular porous structure containing $16(4 \times 4)$ cells, of which only the symmetric quarter are shown here. The ratio of the cell volume to the total volume is 0.0625 . The Von Mises stress distribution over the symmetrical portion of the standard finite element analysis is shown on the left and the Von Mises stress distribution predicted using a homogenization analysis over one cell from the innermost four cells is shown on the right. There is a very close agreement between the homogenization and direct estimate of the stress distribution. 


\section{Experimental evaluation}

The predicted and experimentally measured orthogonal moduli were compared using linear regression of the form $Y=A X+B$, where $Y$ is the predicted modulus and $X$ is the experimental modulus. If the predicted modulus exactly matched the experimental modulus then the regression equation would be $Y=X$ with $R^{2}=1.0$. The results are separated by metaphyseal region for the proximal humerus (Table 1), proximal tibia (Table 2), and distal femur (Table 3).

The best fits between predicted and experimental moduli were found between the strut and the proximal humerus specimens. The coefficients for the regression equation were greater than 0.80 and the constants were less than $30.0 \mathrm{MPa}$ for the AP and ML moduli. The prediction for the IS modulus was not quite as good with a coefficient of 0.60 and a constant of $76 \mathrm{MPa}$ for the strut model. For the distal femur and proximal tibia specimens, the strut model did not predict nearly as well as for the proximal humeri specimens. Although the $R^{2}$ values were greater than 0.5 for the IS and AP moduli, the coefficients were generally under 0.35 and the constants were around
$100 \mathrm{MPa}$. These values indicated that the strut model overpredicted the experimental IS and AP moduli at low bone volume fractions $(<0.15)$ and underpredicted the moduli at higher bone volume fractions $(>0.20)$. The strut model always overpredicted the ML moduli of the distal femur and proximal tibia specimens. While the cubic plate model predictions followed the trend of the strut model, the cubic plate model always overpredicted the modulus values except at the highest bone volume fractions $(>0.30)$.

Changing the structural symmetry of the cells from cubic to orthotropic did not improve the modulus prediction. Although the ratio of the principal axes of the orthotropic cells matched the MIL ratios of the specimens, the predicted orthotropic elastic constants did not differ appreciably from the cubic elastic constants. Therefore, the predictions of the orthotropic models were very similar to the predictions of the cubically symmetric models. In this study, the greatest contribution to the difference between the three predicted moduli (AP, ML, IS), was the orientation of the cells with respect to the anatomic directions, not the principal structural symmetry of the cells.

Table 1. Linear regression between predicted and experimental modulus values for the proximal humerus specimens

\begin{tabular}{llcrcrcr}
\hline $\begin{array}{l}\text { Microstructural } \\
\text { model }\end{array}$ & Modulus & $A$ & $B$ & $R^{2}$ & $p$ & $n \begin{array}{c}\text { Standard error } \\
\text { of estimate }\end{array}$ \\
\hline Cubic strut & AP & 0.83 & 32.0 & 0.77 & $<0.01$ & 9 & 62.1 \\
Cubic strut & ML & 0.86 & -5.7 & 0.79 & $<0.01$ & 9 & 69.6 \\
Cubic strut & IS & 0.59 & 76.2 & 0.53 & $<0.01$ & 9 & 62.2 \\
Orthotropic strut & AP & 0.82 & 30.6 & 0.73 & $<0.01$ & 9 & 67.8 \\
Orthotropic strut & ML & 0.84 & -5.8 & 0.76 & $<0.01$ & 9 & 72.7 \\
Orthotropic strut & IS & 0.55 & 77.7 & 0.43 & $<0.05$ & 9 & 70.4 \\
Cubic plate & AP & 1.21 & 329.6 & 0.90 & $<0.01$ & 9 & 56.7 \\
Cubic plate & ML & 1.21 & 278.2 & 0.75 & $<0.01$ & 9 & 109.2 \\
Cubic plate & IS & 0.96 & 379.8 & 0.65 & $<0.01$ & 9 & 79.3 \\
\hline
\end{tabular}

Coefficients of the regression equation $Y=A X+B$ are presented along with $R^{2}, p$ values, and standard error of estimate. For the modulus, $\mathrm{AP}=$ anterior-posterior, $\mathrm{ML}=$ medial-lateral, $\mathrm{IS}=$ inferior-superior. $B$ and standard error of estimate are in MPa.

Table 2. Linear regression between predicted and experimental modulus values for the proximal tibia specimens

\begin{tabular}{llrrrrrr}
\hline $\begin{array}{l}\text { Microstructural } \\
\text { model }\end{array}$ & Modulus & $A$ & $B$ & $R^{2}$ & $p$ & $n$ & $\begin{array}{c}\text { Standard error } \\
\text { of estimate }\end{array}$ \\
\hline Cubic strut & AP & 0.90 & 56.4 & 0.30 & $<0.10$ & 10 & 120.6 \\
Cubic strut & ML & 0.34 & 113.6 & 0.12 & $<0.35$ & 10 & 104.4 \\
Cubic strut & IS & 0.36 & 104.4 & 0.54 & $<0.05$ & 10 & 91.2 \\
Orthotropic strut & AP & 0.82 & 54.0 & 0.27 & $<0.15$ & 10 & 118.9 \\
Orthotropic strut & ML & 0.27 & 105.4 & 0.09 & $<0.40$ & 10 & 94.2 \\
Orthotropic strut & IS & 0.30 & 82.5 & 0.56 & $<0.01$ & 10 & 74.8 \\
Cubic plate & AP & 1.64 & 292.2 & 0.53 & $<0.05$ & 10 & 136.2 \\
Cubic plate & ML & 0.88 & 391.6 & 0.28 & $<0.15$ & 10 & 155.0 \\
Cubic plate & IS & 0.62 & 354.2 & 0.65 & $<0.01$ & 10 & 126.6 \\
Orthotropic plate & AP & 1.63 & 288.8 & 0.53 & $<0.05$ & 10 & 136.0 \\
Orthotropic plate & ML & 0.88 & 387.6 & 0.28 & $<0.15$ & 10 & 155.7 \\
Orthotropic plate & IS & $\mathbf{0 . 6 3}$ & 362.9 & 0.64 & $<0.01$ & 10 & 131.2 \\
\hline
\end{tabular}

Coefficients of the regression equation $Y=A X+B$ are presented along with $R^{2}, p$ values, and standard error of estimate. For the modulus, $\mathbf{A P}=$ anterior-posterior, $\mathrm{ML}=$ medial-lateral, $\mathrm{IS}=$ inferior-superior. $B$ and standard error of estimate are in $\mathrm{MPa}$. 
Table 3. Linear regression between predicted and experimental modulus values for the distal femur specimens

\begin{tabular}{llccccrr}
\hline $\begin{array}{l}\text { Microstructural } \\
\text { model }\end{array}$ & Modulus & $A$ & $B$ & $R^{2}$ & $p$ & $n$ & $\begin{array}{c}\text { Standard error } \\
\text { of estimate }\end{array}$ \\
\hline Cubic strut & AP & 0.32 & 133.9 & 0.56 & $<0.00$ & 19 & 98.8 \\
Cubic strut & ML & 0.35 & 313.1 & 0.09 & $<0.30$ & 19 & 216.5 \\
Cubic strut & IS & 0.32 & 108.2 & 0.60 & $<0.00$ & 19 & 87.0 \\
Orthotropic strut & AP & 0.27 & 108.0 & 0.52 & $<0.00$ & 19 & 91.1 \\
Orthotropic strut & ML & 0.32 & 318.5 & 0.08 & $<0.30$ & 19 & 217.5 \\
Orthotropic strut & IS & 0.26 & 91.2 & 0.56 & $<0.00$ & 19 & 76.8 \\
Cubic plate & AP & 0.45 & 513.9 & 0.49 & $<0.00$ & 19 & 159.8 \\
Cubic plate & ML & 0.58 & 691.7 & 0.17 & $<0.10$ & 19 & 249.9 \\
Cubic plate & IS & 0.52 & 447.3 & 0.65 & $<0.00$ & 19 & 127.6 \\
Orthotropic plate & AP & 0.51 & 502.9 & 0.50 & $<0.00$ & 19 & 173.6 \\
Orthotropic plate & ML & 0.62 & 671.6 & 0.17 & $<0.10$ & 19 & 262.8 \\
Orthotropic plate & IS & 0.55 & 440.9 & 0.61 & $<0.00$ & 19 & 145.9 \\
\hline
\end{tabular}

Coefficients of the regression equation $Y=A X+B$ are presented along with $R^{2}, p$ values, and standard error of estimate. For the modulus, $\mathrm{AP}=$ anterior-posterior, $\mathrm{ML}=$ medial-lateral, $\mathrm{IS}=$ inferior-superior. $B$ and standard error of estimate are in $\mathrm{MPa}$.
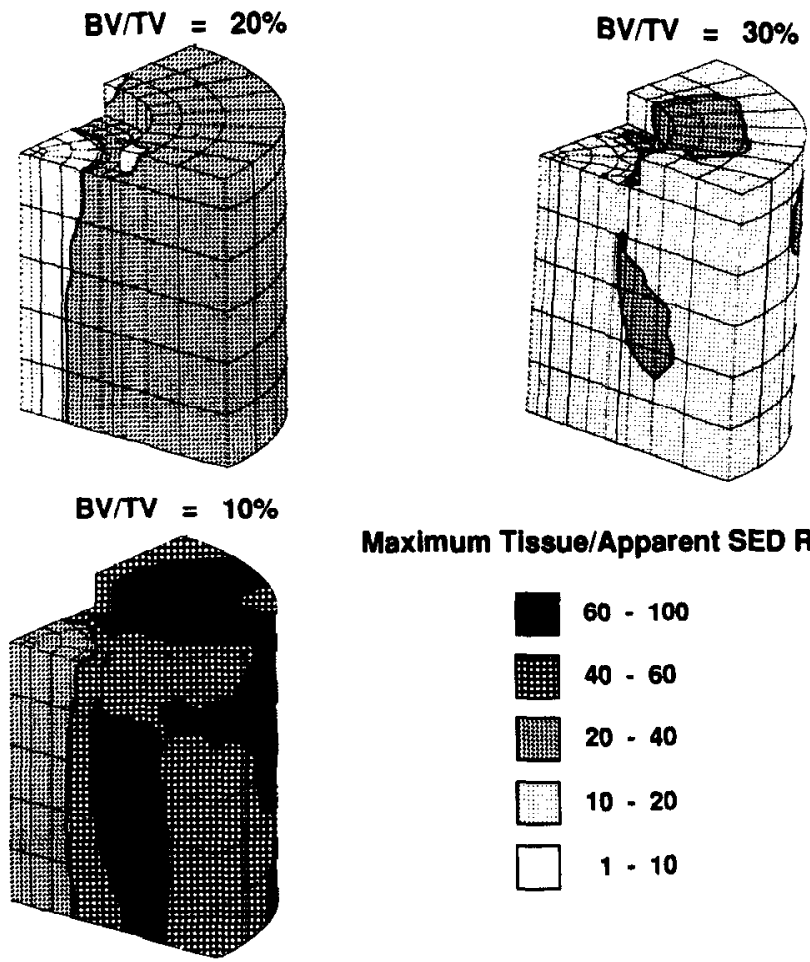

\section{Maximum Tissue/Apparent SED Ratio}

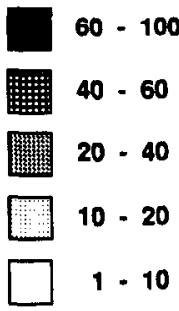

Fig. 7. Ratio of the maximum tissue SED to the apparent SED for a model of trabecular bone containing the strut microstructure. The apparent SED was calculated from the global model with apparent stiffness from the strut microstructure. The maximum tissue SED was then determined from the tissue SED distribution over the microstructural cell at each location in the peg model. Bone volume fractions of 10,20, and $30 \%$ are shown. With increasing volume fraction, the difference between the maximum tissue and apparent SED is reduced.

\section{Comparison of apparent and tissue strain energy density}

To directly compare the tissue and apparent SED, the ratio of the maximum tissue SED to the apparent SED was plotted as a contour for the strut microstructure (Fig. 7), the spherical void microstructure (Fig. 8), and the closed cell microstructure (Fig. 9). Bone volume fractions of 10,20 , and $30 \%$ were analyzed since these fell in the range of volume fractions of the experimental specimens from Section 3 of the results. For the closed cell microstructure, the SED ratio was between 10.0 and 60.0 at $10 \% \mathrm{BV} / \mathrm{TV}$ and decreased to between 1.0 and 10.0 at $30 \% \mathrm{BV} / \mathrm{TV}$. The SED ratio for the strut model was between 30.0 and 60.0 at $10 \% \mathrm{BV} / \mathrm{TV}$ and decreased to between 

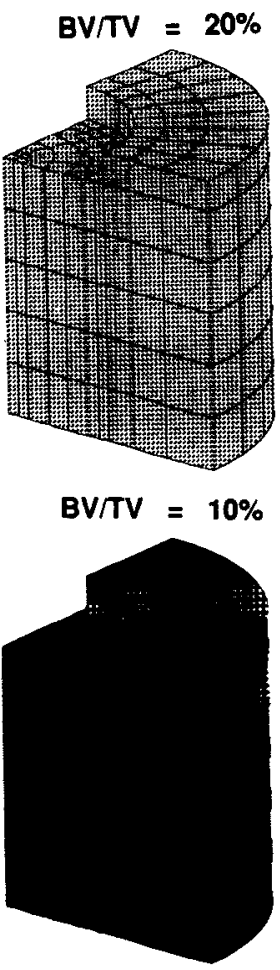

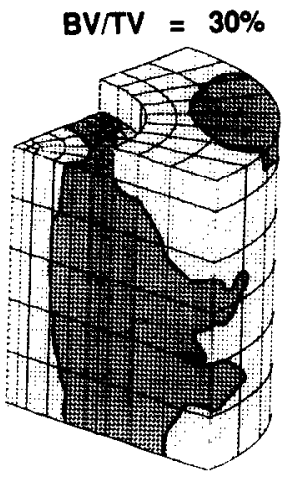

Maximum Tissue/Apparent SED Ratio

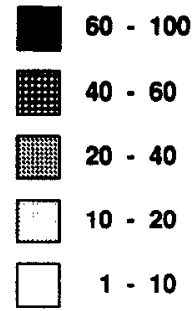

Fig. 8. Ratio of the maximum tissue SED to the apparent SED for a model of trabecular bone containing the spherical void microstructure. The apparent SED was calculated from the global model with apparent stiffness from the spherical void microstructure. The maximum tissue SED was then determined from the tissue SED distribution over the microstructural cell at each location in the peg model. Bone volume fractions of 10,20 , and $30 \%$ are shown. With increasing volume fraction, the difference between the maximum tissue SED and apparent SED is reduced.

10.0 and 30.0 at $30 \% \mathrm{BV} / \mathrm{TV}$. The results for the spherical void model showed the greatest difference between maximum tissue SED and the apparent SED. The spherical void SED ratios were between 30.0 and 120.0 at $10 \% \mathrm{BV} / \mathrm{TV}$ and decreased to between 10.0 and 30.0 at $30 \% \mathrm{BV} / \mathrm{TV}$. Since it is obvious that apparent SED will not represent the true tissue SED, some investigators have suggested using the apparent SED modified by the volume fraction to estimate the tissue SED (Carter et al., 1987; Huiskes et al., 1987). Dividing the apparent SED by the bone volume fraction will accurately predict the average hard tissue SED if two conditions are satisfied: (1) the exact apparent stiffness matrix was used to predict the apparent SED and (2) the stress in the marrow is negligible compared with the hard tissue stress. However, dividing the apparent SED by BV/TV will not give the maximum tissue SED nor will it give the distribution of tissue SED. Comparison of peak tissue SED with apparent SED divided by BV/TV reduced the ratio between the quantities, but the distribution of the ratio did not differ from the original peak tissue/ apparent SED ratio. The modified apparent SED ratio showed the same dependence on microstructural geometry and BV/TV as did the unmodified tissue/apparent SED ratio. Thus, dividing the apparent SED by the BV/TV really represents a scaling of the original difference between tissue and apparent SED. At bone volume fractions near 1.0, the distribution of tissue SED about the mean tissue strain is quite small, making the mean tissue SED a good estimate of the peak value. For lower bone volume fractions, however, the deviation of tissue SED from the mean becomes quite large, and the mean tissue SED is a poor measure of the distribution of tissue SED.

The interaction between amount and type of microstructure was further demonstrated by plotting the ratio between the maximum SED distribution of the strut and the spherical void model (Fig. 10). In this case, the SED in the spherical void microstructure was much greater than the SED in the strut microstructure at $10 \%$ volume fraction, while the SED values at $30 \%$ volume fraction were fairly similar. These results show that predictions of tissue stress are very dependent on the geometry of the microstructure. The dependence of tissue stress on geometry is prevalent at volume fractions less than $20 \%$, suggesting that the structure of individual trabeculae becomes increasingly important in determining mechanical behavior of metaphyseal regions as bone mass is lost.

\section{DISCUSSION}

Many investigators have noted that trabecular bone apparent stiffness and strength are very dependent on 

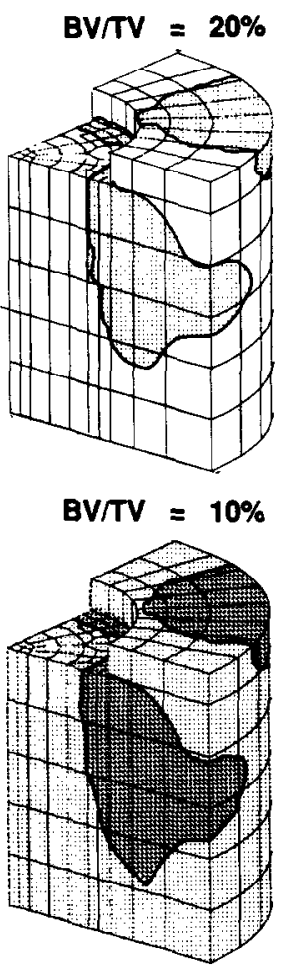

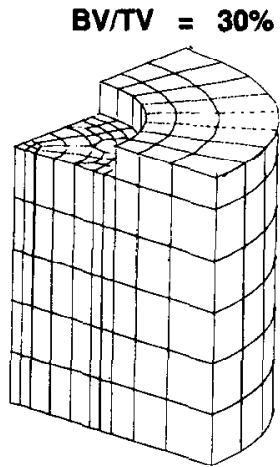

Maximum Tissue/Apparent SED Ratio

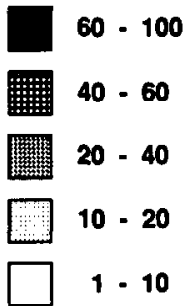

Fig. 9. Ratio of the maximum tissue SED to the apparent SED for a model of trabecular bone containing the closed plate microstructure. The apparent SED was calculated from the global model with apparent stiffness from the closed plate microstructure. The maximum tissue SED was then determined from the tissue SED distribution over the microstructural cell at each location in the peg model. Bone volume fractions of 10,20 and $30 \%$ are shown. With increasing volume fraction, the difference between the maximum tissue SED and apparent SED is reduced.

the trabecular microstructure (Goldstein, 1987; Snyder et al., 1989). Investigators have also stated that tissue stress estimates may be necessary to accurately quantify and predict mechanically adaptive trabecular bone remodeling (Carter et al., 1987; Cheal et al., 1987; Huiskes et al., 1987). These two factors suggest that microstructural analysis is needed to further our understanding of the mechanical and remodeling characteristics of trabecular bone. Previous microstructural analyses of trabecular bone have provided estimates of apparent stiffness and failure stress under assumed boundary conditions (Beaupre and Hayes, 1985; Gibson, 1985; Williams and Lewis, 1981; Pugh et al., 1973). However, no studies in orthopaedic biomechanics have reported estimates of tissue level stress distribution for a large region of trabecular bone. The homogenization theory provides a systematic way to estimate tissue stresses for whole metaphyseal regions. Initial applications to developing stress morphology relationships for bone remodeling (Hollister et al., 1990a), predicting trabecular bone apparent strength (Fyhrie et al., 1989), and developing a bone remodeling theory based on tissue strain (Fyhrie and Hollister, $1990 \mathrm{a}$, b) have recently been presented.

The most significant differences between the homogenization theory and standard mechanics approaches are the assumptions made to calculate the apparent stiffness and the tissue strain from the apparent strain. By separating the tissue strain into two components, and by assuming the microstructure is periodic, the homogenization theory provides a mathematically rigorous basis for decoupling microstructural level analyses from continuum or apparent level analyses (Bensoussan et al., 1978; Sanchez-Palencia, 1980; refer to Appendix A for a brief discussion). If the microstructure is periodic, the homogenization theory will give nearly exact estimates of the tissue level strain independent of the apparent strain. If the microstructure is non-periodic, the homogenization theory will give tissue strain estimates whose error is related to the degree of structural non-periodicity (Hollister $e t$ al., 1990b). Thus, it may be possible to construct error bounds for analyzing non-periodic materials, like trabecular bone, with the homogenization theory based on the degree of structural non-periodicity.

Standard mechanics approaches approximate either the apparent strain, $\varepsilon_{a p p}$, by displacement boundary conditions (Christenson, 1986; Beaupre and Hayes, 1985; Iremonger and Lawler, 1980; Hill, 1963; Hashin, 1962), or the apparent stress, $\sigma_{\text {app }}$, by traction boundary conditions (Gibson and Ashby, 1988; Huber and Gibson, 1988; Gibson and Ashby, 1982; Kanakkanatt, 1973; Patel and Finnie, 1970; Ko, 1965; Gent and Thomas, 1959). These approaches then solve for 

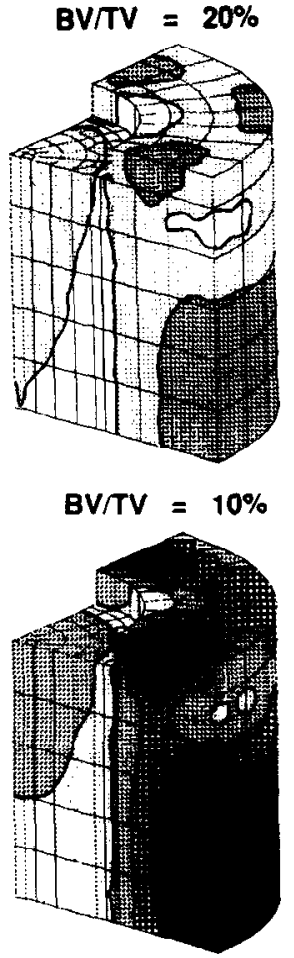

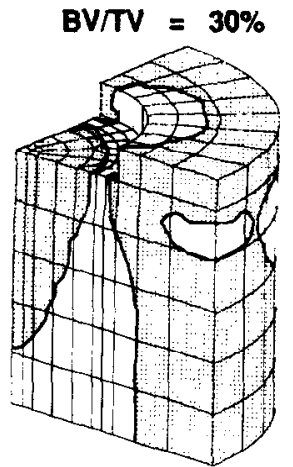

Spherical Void/Strut SED Ratio

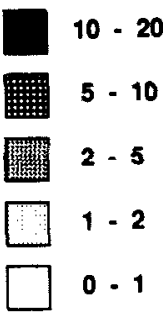

Fig. 10. Ratio of the spherical void maximum tissue SED to the strut maximum tissue SED for bone volume fractions of 10,20 and $30 \%$ for model of a metal peg loading a large region of trabecular bone. The maximum tissue SED predicted by the spherical void microstructure is much larger than the SED predicted by the strut microstructure, especially at bone volume fractions below $20 \%$. This indicates the influence of trabecular bone microstructural geometry on the tissue level stress and strain.

the total tissue strain using the assumed boundary conditions. Applying displacement boundary conditions will give an upper bound on the apparent stiffness while traction boundary conditions will give a lower bound (Hill, 1963; Hashin, 1962; Suquet, 1985). Physically, this means that the average of the tissue stresses may not be in equilibrium with an arbitrary apparent stress when applied displacements are assumed, or conversely, that the average of the tissue strains may not be compatible with an arbitrary apparent strain when applied tractions are assumed. This was demonstrated by Beaupre and Hayes (1985) who found that a linear shear strain displacement boundary condition produced a stress distribution 16 times greater than that required to satisfy equilibrium. Although Beaupre and Hayes found better agreement with a nonlinear boundary condition, this boundary condition was estimated by an auxiliary two-dimensional analysis of 16 cells ( 4 cells on a side).

The results of both Beaupre and Hayes (1985) and Hill (1963) show that it is necessary to increase the number of cells analyzed using standard mechanics approaches to produce better apparent stiffness estimates for a given microstructure. Because in situ cell boundary conditions are not known a priori, most investigators have applied either uniform traction or uniform displacements to the cell boundary (Gibson and Ashby, 1988; Huber and Gibson, 1988; Hashin,
1983; Iremonger and Lawler, 1980; Kanakkanatt, 1973; Patel and Finnie, 1970; Ko, 1965). Hollister et al. (1990b) found that errors in tissue strain energy distribution were unrelated to the degree of structural periodicity when uniform displacement or traction boundary conditions were used for microstructural analysis. Thus, unlike the homogenization theory, it may not be possible to estimate errors in tissue stress or strain approximations for standard mechanics approaches based on the degree of structural nonperiodicity.

Homogenization predictions of apparent stiffness for a porous composite were between analytical predictions using uniform traction and uniform displacement boundary conditions for volume fractions greater than 0.20 . At volume fractions less than 0.20 , the homogenization finite element approximations were slightly higher $(5 \%)$ than the upper bound. Use of a selective reduced integration scheme for the finite element solution produced apparent stiffness predictions equal to the predictions of the uniform displacement boundary conditions. This result suggests that selective reduced integration techniques should be applied when modeling microstructures where the thickness in one direction is one-tenth or less than the thickness of the other two directions. Since the experimental results presented by Ishai and Cohen were either between the analytical bounds (volume fraction 
$>0.70$ ) or close to the analytical bounds (volume fraction $<0.70$ ), this shows that the homogenization theory can accurately predict the apparent stiffness of regular porous composites.

Strut and closed cell models of trabecular bone microstructure, which matched BV/TV, BS/BV, and trabecular orientation predicted experimentally measured trabecular bone stiffness with reasonable accuracy, depending on metaphyseal region. Modeling trabecular orientation was very important for accurate predictions. If orientation was not included in the model, the experimental elastic moduli were almost always overestimated by $200-300 \mathrm{MPa}$ for the strut model. Modifying the axes ratio of the cubically symmetric models to match the Mean Intercept Length (MIL) ratios of the specimens did not improve the accuracy of the predictions. This result, along with overprediction by models which did not include orientation, suggests that the trabecular orientation is a major contributing factor to large moduli ratios measured in specimens aligned with anatomic axes (Ciarelli et al., 1986).

Another factor affecting the accuracy of microstructural models is the variance of trabecular geometry between different metaphyseal regions and also within a metaphyseal region. The differences between model prediction accuracy for the proximal humerus, distal femur, and proximal tibia suggests that different microstructural models may be needed for each metaphyseal region analyzed. In our laboratory, we have found that MIL ratios for the proximal humerus are much less than those in the distal femur or proximal tibia. The low MIL ratios of the proximal humerus suggest that trabecular bone of the proximal humerus is a much more equiaxial structure than that in the distal femur or proximal tibia. This may explain why the cubically symmetric models predicted the stiffness of the proximal humeri specimens much better than that of the distal femur and proximal tibia. The MIL ratio may therefore give some information as to the proper shape to model. For instance, a large MIL ratio may indicate more of a plate-strut structure than a pure equiaxial strut structure. These different types of structures have been observed in different metaphyseal regions (Singh, 1978; Parfitt et al., 1983; Gibson, 1985; Feldkamp et al., 1989).

Relationships between maximum tissue stress quantities and apparent stress quantities are a complex function of both the amount and shape of the trabecular tissue. Dividing the apparent SED by BV/TV may not adequately predict peak tissue SED since it is an estimation of the mean tissue SED. The mean tissue SED is a good estimate of the peak SED value if the deviation of tissue SED about the mean is small, which occurs when the bone volume fraction is near one (cortical bone). For the models used in this paper, the deviation of tissue SED from the mean was a strong function of $\mathrm{BV} / \mathrm{TV}$ and the microstructural geometry. In particular, for BV/TV less than 0.3 , the mean tissue SED for the open celled models was an order of magnitude incorrect in predicting the peak.

\section{CONCLUSIONS}

The homogenization theory provides the ability to directly estimate microstructural stress and strain variations from standard finite element apparent strain estimates. This method provides a rigorous mathematical basis for decoupling the tissue and apparent level analyses by separating the tissue strain into an apparent and fluctuating component. The resulting formulation differs from traditional mechanics approaches to microstructural analysis. For problems within the realm of its assumptions, the homogenization theory provides very accurate estimates of both apparent stiffness and local strain for regular porous structures.

Improvements in modeling trabecular bone mechanics using the homogenization theory depend on the development of more accurate models of trabecular bone structure and on determining the periodicity of trabecular bone microstructure. Initial experimental evaluation of microstructural models suggests that different microstructural models may be needed for different metaphyseal regions, as well as for different volume fractions of trabecular bone within a metaphyseal region. Additionally, the possibility of a trabecular tissue modulus size effect (Choi et al., 1990) needs to be examined in future microstructural models of trabecular bone.

Current studies are addressing, in a more rigorous manner, the effect of periodic boundary conditions, cell size ratio assumptions on accuracy when modeling both periodic and non-periodic microstructures and development of a generally valid cellular model. The results of these studies and their implications for modeling trabecular bone will be reported at a later date. In conclusion, the homogenization theory should be further investigated as a means for analyzing trabecular bone mechanics, and possibly the mechanics of other biological tissues as well.

Acknowledgements-The authors would like to thank professor N. Kikuchi and Dr J. Guedes for the many discussions of homogenization theory. We would also like to thank Mr E. Kessler and Ms Wanchuan Kao for running some finite element analyses. Supported by NIH (AR34399, AR31793).

\section{REFERENCES}

Beaupre, G. S. and Hayes, W. C. (1985) Finite element analysis of a three-dimensional open-celled model for trabecular bone. J. biomech. Engng 107, 249-256.

Bendsoe, M. P. and Kikuchi, N. (1988) Generating optimal topologies in structural design using a homogenization method. Commun. Meth. appl. Mech. Engng 71, 192-224.

Bensoussan, A., Lions, J. L. and Papanicolaou, G. (1978) Asymptotic Analysis for Periodic Structures. North. Holland, Amsterdam.

Carter, D. R., Fyhrie, D. P. and Whalen, R. T. (1987) Trabecular bone density and loading history: regulation of 
connective tissue biology by mechanical energy. $J$. Biomechanics 20, 785-795.

Cheal, E. J., Snyder, B. D., Nunamaker, D. M. and Hayes, W C. (1987) Trabecular bone remodeling around smooth and porous implants in an equine patellar model. $J$. Biomechanics 20, 1121-1134.

Choi, K., Kuhn, J. L., Ciarelli, M. J. and Goldstein, S. A (1990) The elastic modulus of trabecular, subchondral, and cortical bone tissue and the size-dependency of cortical bone modulus. J. Biomechanics 23, 1103-1113.

Christenson, R. M. (1986) Mechanics of low density materials. J. Mech. Phys. Solids 34, 563-578.

Ciarelli, M. J., Goldstein, S. A., Dickie, D., Ku, J. L., Kapper, M., Stanley, J., Flynn, M. J. and Matthews, L. S. (1986) Experimental determination of the orthogonal mechanical properties, density, and distribution of human trabecular bone from the major metaphyseal regions utilizing materials testing and computed tomography. Trans. 32nd $A$. Meeting Orthop. Res. Soc. p. 42. ORS, New Orleans.

Counto, U. J. (1964) The effect of the elastic modulus of the aggregate on the elastic modulus, creep, and creep recovery of concrete. Mag. Concr. Res. 16, 129-138.

Crolet, J. M., Aoubiza, B. and Meunier, A. (1988) A numerical model of anisotropic elastic properties of osteons. Proceedings 12th Annual Meeting American Society of Biomechanics, University of Illinois at Urbana, Champaign, pp. $177-178$.

Crolet, J. M. (1990) Homogenization: mathematical method applied to haversian cortical bone structure. Proceedings lst World Congress of Biomechanics, p. 156.

Feldkamp, L. A., Goldstein, S. A., Parfitt, A. M., Jesion, G. and Kleerekoper, M. (1989) The direct examination of three-dimensional bone architecture in vitro by computed tomography. J. Bone Min. Res. 4, 3-11.

Fyhrie, D. P., Jepsen, K. J., Hollister, S. J. and Goldstein, S. A. (1989) Predicting trabecular bone strength and microstrains using homogenization theory: application to a vertebra. Proceedings of the XIIth International Congress of Biomechanics, pp. 173-174.

Fyhrie, D. P. and Hollister, S. J. (1990a) A tissue strain remodeling theory for trabecular bone using homogenization theory. Trans. 36th A. Meeting Orthop. Res. Soc. p. 76. ORS, Las Vegas.

Fyhrie, D. P. and Hollister, S. J. (1990b) Comparison of a trabecular tissue strain remodeling theory to experimental results. Trans. 36th A. Meeting Orthop. Res. Soc. p. 107. ORS, Las Vegas.

Gent, A. N. and Thomas, A. G. (1959) The deformation of foamed elastic materials. J. appl. Polymer Sci. 1, 107-113.

Gibson, L. J. (1985) The mechanical behavior of cancellous bone. J. Biomechanics 18, 317-328.

Gibson, L. J. and Ashby, M. F. (1982) The mechanics of threedimensional cellular materials. Proc. R. Soc. Lond. A382, 43-59.

Gibson, L. J. and Ashby, M. F. (1988) Cellular Solids: Structure and Properties. Pergamon Press, Oxford.

Goldstein, S. A. (1987) The mechanical properties of trabecular bone: dependence on anatomic location and function. J. Biomechanics 20, 1055-1062.

Goulet, R. W., Feldkamp, L. A., Kubinski, D. J. and Goldstein, S. A. (1989) Predicting the architectural orientation of trabecular bone. Trans. 35th A. Meeting Orthop. Res. Soc. p. 263. ORS, Las Vegas.

Guedes, J. M. (1990) Nonlinear computational models for composite materials using homogenization. Ph.D. dissertation, University of Michigan.

Hashin, Z. (1962) The tastic moduli of heterogeneous materials. J. appl. Mech. 29, 143-150.

Hashin, Z. (1983) Analysis of composite materials - a survey. J. appl. Mech. 50, 481-505.

Hill, R. (1963) Elastic properties of reinforced solids: some theoretical principles. J. Mech. Phys. Solids 11, 357-372.

Hilyard, N. C. (Ed.) (1982) Mechanics of Cellular Plastics.
Macmillan, New York.

Hollister, S. J., Fyhrie, D. P., Jepsen, K. J. and Goldstein, S. A (1989) An analysis of trabecular bone micro-mechanics using homogenization theory with comparison to experimental results. Proceedings of the XIIth International Congress of Biomechanics, pp. 110-111.

Hollister, S. J., Goldstein, S. A., Jepsen, K. J. and Goulet, R. W. (1990a) Continuum and microstructural stress morphology relationships for trabecular bone subject to controlled implant loads. Trans. 36th ORS, p. 74.

Hollister, S. J., Kikuchi, N., Borodkin, J. L. and Goldstein, S. A. (1990b) A study of microstructural analysis methods: implications for modeling trabecular bone microstructure In 1990 Advances in Bioengineering (Edited by Goldstein, S. A.) ASME BED-Vol. 17, 387-390.

Huber, A. T. and Gibson, L. J. (1988) Anisotropy of foams. J. Mater. Sci. 23, 3031-3040.

Huiskes, R. and Chao, E. Y. S. (1983) A survey of finite element analysis in orthopedic biomechanics: the first decade. $J$. Biomechanics 16, 385-410.

Huiskes, R., Weinans, H., Grootenboer, H. J., Dalstra, M., rudala, B. and Slooff, T. J. (1987) Adaptive boneremodeling applied to prosthetic-design analysis. J. Biomechanics 20, 1135-1150.

Iremonger, M. J. and Lawler, J. P. (1980) Relationship between modulus and density for high-density closed cell thermoplastic foams. J. appl. Polymer Sci. 25, 809-819.

Ishai, O. (1965) Contribution (response to U. J. Counto, Mag. Concr. Res., 1964). Mag. Concr. Res. 17, 148-149.

Ishai, O. and Cohen, L. J. (1967) Elastic properties of filled and porous epoxy composites. Int. J. Mech. Sci. 9, 539-546.

Kanakkanatt, S. V. (1973) Mechanical anisotropy of opencelled foams. J. Cell. Plastics 9, 50-53.

Kikuchi, N. (1986) Finite Element Methods in Mechanics. Cambridge University Press, Cambridge.

Ko, W. L. (1965) Deformations of foamed elastomers. J. Cell. Plastics 1, 45-50.

Kuhn, J. L., Goldstein, S. A., Choi, K., London, M., Feldkamp, L. A. and Matthews, L. S. (1989) A comparison of the trabecular and cortical tissue moduli from human iliac crests. J. Orthop. Res. 7, 876-884.

Lederman, J. M. (1971) The prediction of the tensile properties of flexible foams. J. appl. Polymer Sci. 15, 693-703.

Lurie, K. A., Fedorov, A. V. and Cherkaev, A. V. (1982) Regularization of optimal design problems for bars and plates, I and II. J. Opt. Theory App. 37, 499-543.

Luxmoore, A. R. and Owens, D. R. J. (1982) Syntatic foams. In Mechanics of Cellular Plastics (Edited by Hilyard, N.). Macmillan, New York.

McElhaney, J., Alem, N. and Roberts, V. (1970) $A$ porous block model for cancellous bones, ASME Publication No. 70-WA/BHF-2, 1-9.

Mente, P. L. and Lewis, J. L. (1989) Experimental method for the measurement of the elastic modulus of trabecular bone tissue. J. Orthop. Res. 7, 456-461.

Parfitt, A. M., Mathews, C. H. E., Villanueva, A. R., Kleerekoper, M., Frame, B. and Rao, D. S. (1983) Relationships between surface, volume, and thickness of iliac trabecular bone in aging and in osteoporosis: implications for the microanatomic and cellular mechanism of bone loss. $J$. clin. Invest. 72, 1396-1409.

Patel, M. R. and Finnie, I. (1970) Structural features and mechanical properties of rigid cellular plastics. J. Materials 5, 909-932.

Pugh, J. W., Rose, R. M. and Radin, E. L. (1973) A structural model for the mechanical behavior of trabecular bone. J. Biomechanics 6, 657-670.

Sanchez-Palencia, E. (1980) Non-Homogeneous Media and Vibration Theory. Springer, Berlin.

Singh, I. (1978) The architecture of cancellous bone. J. Anat. 127, 305-310

Snyder, B. D., Cheal, F. J., Hipp, J. A and Hayes, W. C. (1989) 
Anisotropic structure-property relations for trabecular bone. Trans. 35th A. Meeting Orthop. Res. Soc. p. 265. ORS, Las Vegas, Nevada.

Suquet, P. M. (1985) Elements of homogenization theory for inelastic solid mechanics. In Homogenization Techniques for Composite Media (Edited by Sanchez-Palencia, E. and Zaoui, A.). Springer, Berlin.

Williams, J. L. and Lewis, J. L. (1982) Properties and an anisotropic model of cancellous bone from the proximal tibia epiphysis. J. biomech. Engng 104, 50-56.

\section{APPENDIX A}

\section{Derivation of the homogenization elasticity equations}

In homogenization theory, the total microstructural displacement is expanded asymptotically:

$$
\{u(x, y)\}=\left\{u_{0}(x)\right\}+\eta\left\{u_{1}(x, y)\right\}+\eta^{2}\left\{u_{z}(x, y)\right\}+\ldots,
$$

where $u$ is the total microstructural displacement, $u_{0}$ is the apparent level displacement, $u_{i}$ are perturbations in the displacement field due to the microstructure, $x$ is the apparent level coordinate system, $y$ is the microstructural level coordinate system, and $\eta$ is the ratio of the microstructure size to the region in which it exists. It is important to note that $u_{0}$ is only a function of $x$ which implies that the microstructure is locally periodic. This means that the microstructural geometry may vary at different points on the apparent or continuum level. The coordinates $x$ and $y$ are related by:

$$
y=\frac{x}{\eta} .
$$

Physically, equation (A2) means that quantities like stress or strain vary $1 / \eta$ times more rapidly on the microstructural level than on the apparent level. Derivatives with respect to $x$ are written using the chain rule as:

$$
\frac{\partial}{\partial x}=\frac{\partial}{\partial x}+\frac{1}{\eta} \frac{\partial}{\partial y}
$$

The small deformation strain vector is written using equation (A1) as:

$$
\begin{aligned}
\{\varepsilon(u)\}= & \frac{1}{2}\left(\frac{\partial u_{i}}{\partial x_{j}}+\frac{\partial u_{j}}{\partial x_{i}}\right)=\frac{1}{2}\left[\left(\frac{\partial u_{0 i}}{\partial x_{j}}+\frac{\partial u_{0 j}}{\partial x_{i}}\right)\right. \\
& \left.+\left(\frac{\partial u_{1 i}}{\partial y_{j}}+\frac{\partial u_{1 j}}{\partial y_{i}}\right)+\eta\left(\frac{\partial u_{1 i}}{\partial x_{j}}+\frac{\partial u_{i j}}{\partial x_{i}}\right)\right]+\ldots
\end{aligned}
$$

If terms of order $O(\eta)$ and higher are neglected the smail strain vector may be written as:

$$
\begin{aligned}
\left\{\varepsilon_{\mathrm{tissue}}\right\} & =\{\varepsilon(u)\}=\left\{\varepsilon_{\mathrm{app}}\right\}+\left\{\varepsilon^{*}\right\} \\
\left\{\varepsilon_{\mathrm{app}}\right\} & =\frac{1}{2}\left(\frac{\partial u_{0 i}}{\partial x_{j}}+\frac{\partial u_{0 j}}{\partial x_{i}}\right) \\
\left\{\varepsilon^{*}\right\} & =\frac{1}{2}\left(\frac{\partial u_{1 t}}{\partial y_{j}}+\frac{\partial u_{1 j}}{\partial y_{i}}\right),
\end{aligned}
$$

where $\{\varepsilon(u)\}$ has been denoted as $\left\{\varepsilon_{\text {tissure }}\right\},\left\{\varepsilon_{\mathrm{app}}\right\}$ is the apparent level strain (calculated by standard continuum finite element programs), and $\left\{\varepsilon^{*}\right\}$ is the fluctuating strain vector (Suquet, 1985). For the virtual work formulation, the virtual strain vector is written as:

$$
\{\varepsilon(v)\}=\frac{1}{2}\left[\left(\frac{\partial v_{i}}{\partial x_{j}}+\frac{\partial v_{j}}{\partial x_{i}}\right)+\left(\frac{\partial v_{i}}{\partial y_{j}}+\frac{\partial v_{j}}{\partial y_{i}}\right)\right] .
$$

The virtual work (or weak form) of the equilibrium equations may be written as:

$$
\int_{\Omega}\{\varepsilon(v)\}^{T}[C]\left\{\varepsilon_{\text {nimuc }}\right\} d \Omega^{\eta}=\int_{\Gamma}\{v\}^{T}\{t\} d \Gamma,
$$

where $\{v\}$ is the virtual strain vector and $\{t\}$ is an applied traction. The traction is assumed to be applied only to the continuum boundary. Assuming the microstructure to be periodic allows the integral in equation (A6) to be separated into microstructure and apparent levels:

$$
\lim _{n \rightarrow 0} \int_{\Omega^{n}} \mathrm{~d} \Omega^{n}=\int_{\Omega} \frac{1}{V_{\text {cell }}} \int_{V_{\text {cell }}} \mathrm{d} V_{\text {cell }} \mathrm{d} \Omega .
$$

Choosing the virtual displacement $v$ to be a function of $y$ only gives:

$$
\int_{\Omega} \frac{1}{V_{\text {eell }}} \int_{V_{\text {cell }}}\{\varepsilon(v)\}^{T}[C]\left\{\left\{\varepsilon_{\mathrm{spp}}\right\}+\left\{\varepsilon^{*}\right\}\right) \mathrm{d} V_{\text {eetj }} \mathrm{d} \Omega=0,
$$

where the right-hand side is zero because there is no traction on the microstructural level. Equation (A8) will be satisfied if the interior intergral is zero which leads to equation given in the text:

$$
\begin{aligned}
\int_{V_{\text {coll }}}\{\varepsilon(v)\}^{T}[C]\left\{\varepsilon^{*}\right\} \mathrm{d} V_{\text {cell }} \\
=-\int_{V_{\text {cesll }}}\{\varepsilon(v)\}^{T}[C]\left\{\varepsilon_{\text {app }}\right\} \mathrm{d} V_{\text {cell }} .
\end{aligned}
$$

Equation (A9) is really a set of three equations where $\left\{\varepsilon_{\mathrm{app}}\right\}$ can be taken out of the integral since it is a function of $x$ only. Equivalently, $\left\{\varepsilon_{\text {app }}\right\}$ may be taken to be unit $x$ normal, unit $y$ normal, and unit $x y$ shear strains (taken as negative by convention; for three-dimensional analysis, include unit $z$ normal, $x z$ shear, and $y z$ shear strains). Doing this gives a set of three equations:

$$
\begin{aligned}
\int_{V_{c o i t}}\{\varepsilon(v)\}^{T}[C] & \left\{\varepsilon^{k *}\right\} d V_{c e l l} \\
= & \int_{V_{c o s i 1}}\{\varepsilon(v)\}^{T}\left\{C^{k}\right\} d V_{\text {cell }}
\end{aligned}
$$

which leads to the following relation between the tissue and apparent strains:

$$
\left\{\varepsilon_{\text {qusauc }}\right\}=[M]\left\{\varepsilon_{\text {app }}\right\} .
$$

Next, choosing the virtual displacement $v$ to be a function of $x$ only, and using equation (A11) in equation (A6) gives:

$$
\begin{gathered}
\int_{\Omega} \frac{1}{V_{\text {cell }}} \int_{V_{\text {coll }}}\{\varepsilon(v)\}^{T}[C][M]\left\{\varepsilon_{\text {app }}\right\} \mathrm{d} V_{\text {cell }} \mathrm{d} \Omega \\
=\int_{\Gamma}\{v\}^{T}\{t\} \mathrm{d} \Gamma .
\end{gathered}
$$

Since both $\{\varepsilon(v)\}$ and $\left\{\varepsilon_{\mathrm{ppp}}\right\}$ are only functions of $x$, they may be removed from the interior integral to give:

$$
\begin{gathered}
\int_{\Omega}\{\varepsilon(v)\}^{T} \frac{1}{V_{\text {cell }}} \int_{V_{\text {cell }}}[C][M] \mathrm{d} V_{\text {cell }}\left\{\varepsilon_{\text {app }}\right\} \mathrm{d} \Omega \\
=\int_{\Gamma}\{v\}^{T}\{t\} \mathrm{d} \Gamma
\end{gathered}
$$

which is the variational equilibrium equation at the apparent or continuum level. The interior integral is then the apparent stifiness:

$$
\left[C_{\mathrm{app}}\right]=\frac{1}{V_{\text {call }}} \int_{\mathrm{V}_{\mathrm{es} \text { II }}}[C][M] \mathrm{d} V_{\text {cell }}
$$

\section{APPENDIX B}

\section{Size-morphology equations}

The equations which relate morphological parameters of bone volume fraction ( $\mathrm{BV} / \mathrm{TV}$ ) and bone surface to bone volume (BS/BV) to the cell outer dimension $A_{0}$ and inner void length $A$ are presented for both open and closed cells. 
Open cell equations:

$$
\mathrm{BV} / \mathrm{TV}=D_{1}+D_{2} *\left(\frac{A}{A_{0}}\right)^{3}-D_{3} *\left(\frac{A}{A_{0}}\right)^{2}
$$

$$
\begin{aligned}
\mathrm{BV} / \mathrm{TV} * \mathrm{BS} / \mathrm{BV}= & D_{4} *\left(\frac{R_{1}}{R_{2}}+\frac{R_{1}}{R_{3}}+1\right) * \frac{A}{A_{0}} \\
& -D_{5} *\left(\frac{R_{1}}{R_{2}}+\frac{R_{1}}{R_{3}}+1\right) *\left(\frac{A}{A_{0}}\right)^{2},
\end{aligned}
$$

where $D_{1}-D_{5}$ are constants reflecting the inner void shape and the ratios $R_{1} / R_{2}$ and $R_{1} / R_{3}$ are the ratios of the first axis cell length to the second and third axis cell lengths, respectively. If these ratios are one, then the cell will have cubic symmetry.
Closed cell equations

$$
\begin{gathered}
\mathrm{BV} / \mathrm{TV}=D_{1}-D_{2} *\left(\frac{A}{A_{0}}\right)^{3} \\
\mathrm{BV} / \mathrm{TV} * \mathrm{BS} / \mathrm{BV}=D_{3} *\left(\frac{R_{1}}{R_{2}}+\frac{R_{1}}{R_{3}}+1\right) *\left(\frac{A}{A_{0}}\right)^{2},
\end{gathered}
$$

where $D_{1}-D_{3}$ are constants reflecting the inner void shape and the other terms are defined in equations (B1) and (B2).

The two sets of equations, either (B1) and (B2), or (B3) and (B4), may be solved simultaneously using Newton's method to determine the correct cell dimensions for a given model to match the parameters BV/TV and BS/BV of a given trabecular bone specimen. 